\title{
Correction pour la mesure de la radioactivité alpha des aérosols atmosphériques prélevés sur filtre
}

\author{
J. LEMAIRE ${ }^{1}$, J.P. LECOURT ${ }^{1}$, C. RIVIER ${ }^{1}$
}

(Article reçu le 18 avril 2008, accepté le 15 juillet 2008)

RÉSUMÉ La mesure de la radioactivité atmosphérique constitue une difficulté métrologique majeure. En fonction des mécanismes de prélèvement sur filtre et des paramètres de mesure, l'activité alpha des aérosols est sous estimée. L'énergie cinétique des particules alpha est atténuée dans la matière qu'elles traversent, et une partie de cette énergie ne parvient plus au détecteur. Le rendement de mesure est alors dégradé et nécessite une correction. Pour répondre aux conditions de mesures usuelles, le laboratoire a développé un modèle empirique pour l'évaluation des corrections de l'activité alpha en fonction de la masse des aérosols déposés sur le filtre. Le modèle a été validé expérimentalement et a permis une meilleure estimation de l'incertitude sur la mesure de l'activité alpha des aérosols. L'étude a contribué à une première évaluation du facteur de correction pour des filtres peu encrassés provenant de la région parisienne : $F_{\alpha}^{\text {labo }}=1,6 \pm 0,5$.

ABSTRACT Correction to the alpha radioactivity measurement from an atmospheric aerosol in a sampling filter.

The measurement for the atmospheric radioactivity constitutes a major metrological issue. According to penetration mechanisms on filter and measurement parameters, the alpha activity of aerosols is underestimated. The kinetic energy of alpha particles is attenuated in the crossed material, and a part of this energy does not reach the detector. The measurement efficiency decreases and requires a correction. The laboratory developed an empirical model for the activity corrections evaluation from the mass of aerosols on the filter. The model was experimentally validated and it gave a better estimation of the uncertainty on the alpha activity measurement of aerosols. The study contributed to a first correction factor evaluation for low clogged filters coming from the Paris region : $F_{\alpha}^{\text {labo }}=1.6 \pm 0.5$.

Keywords: attenuation / alpha radioactivity / aerosol / radioactivity measurement / filter

\section{Introduction}

Le respect de la qualité de l'environnement de l'homme est une exigence de société. Le Commissariat à l'énergie atomique (CEA) place au cœur de sa politique de sécurité la protection de l'environnement, qui se fonde sur la maîtrise des risques inhérents aux activités de recherche, de développement et de mise à

1 CEA, DSV/USLT/SPRE/L2SE/LESE, 18 route du panarama, BP 6, 92265 Fontenay-aux-Roses Cedex, France. 
l'arrêt de ses installations. Elle vise à réduire autant que possible, au regard des impératifs techniques et économiques, l'impact de ses activités sur l'Homme et son environnement.

La réalisation de ces objectifs repose sur un contrôle continu de l'activité des effluents rejetés dans l'environnement et une surveillance de l'environnement des sites. Ces rejets sont soumis à une réglementation générale et à une réglementation spécifique à chacun des sites comportant des installations nucléaires, dans lesquelles sont définies les limites annuelles autorisées pour les rejets ainsi que les modalités de surveillance de l'environnement.

Afin de vérifier que l'impact des rejets sur l'environnement reste négligeable, une surveillance de l'environnement autour des sites est réalisée tout au long de l'année. Elle comprend un suivi de l'exposition gamma ambiante et des mesures, sur des prélèvements représentatifs, de l'activité des poussières atmosphériques, des eaux ainsi que de la chaîne alimentaire. La surveillance des rejets et de l'environnement est assurée par les unités de protection contre les rayonnements (SPRE) de chaque site. Elles rendent compte de leurs mesures aux autorités de tutelle en charge de la santé, de l'industrie et de l'environnement.

Comme dans tous les centres du CEA, celui de Fontenay-aux-Roses assure une surveillance systématique du site et de son environnement et un contrôle continu de ses rejets. En particulier, la surveillance atmosphérique est réalisée par la mesure de l'activité des aérosols prélevés en continu sur des filtres dans plusieurs stations de contrôle. Celle-ci est effectuée à partir d'une chambre de comptage disposée sur l'appareil de prélèvement (BFSAB). Le laboratoire du SPRE est ensuite chargé de la vérifier et de la valider, au moyen d'appareils de mesure de sensibilité plus importante.

Cependant, la mesure de l'activité déposée sur les filtres demeure une difficulté majeure au regard des conditions de prélèvement des aérosols et de leur détection ; en effet, les laboratoires ne disposent pas de filtres étalons suffisamment représentatifs des aérosols collectés permettant le raccordement de leur mesure à une référence primaire. Cette contrainte peut conduire à sous estimer l'activité d'un facteur 2 en fonction du rendement de détection et des corrections de mesure choisies (Grivaud et Pagliardini, 2004).

Dans la pratique, les laboratoires appliquent une correction de l'activité mesurée pour tenir compte des mécanismes de dépôt des aérosols sur les filtres et de leur influence sur la mesure. Cette correction est généralement obtenue à partir de la mesure de sources préparées par dépôts de traceurs radioactifs d'activité connue sur un filtre. Cependant, cette méthode empirique reste insuffisante pour la 
détermination de l'activité alpha des aérosols. En fonction des conditions de prélèvements, les rendements de détection sont dégradés et varient intrinsèquement avec les mécanismes de dépôt des aérosols et les phénomènes d'interaction des particules alpha avec la matière. La prise en compte de ces paramètres dans la mesure globale de l'activité alpha des aérosols nécessite de conduire une étude permettant de modéliser l'évolution du terme correctif en fonction de la pénétration des aérosols dans le medium filtrant et de la densité surfacique du dépôt.

Les principaux paramètres participant à cette modélisation ont été inventoriés puis combinés afin de définir une relation simple entre la masse d'aérosols collectés et l'atténuation de l'énergie des particules alpha dans le filtre.

Après la vérification expérimentale du modèle, l'influence des paramètres d'entrée pour le calcul du facteur d'atténuation a été étudiée afin d'estimer les valeurs « enveloppes » de la correction d'activité la mieux appropriée aux mesures des aérosols collectés par le laboratoire. Cette approche théorique est en cours d'exploitation au laboratoire du SPRE de Fontenay-aux-Roses et est exposée dans cet article.

\section{2. Évaluation des corrections de mesure}

\subsection{Contexte et hypothèses préalables}

En fonction de l'enfouissement des aérosols dans le filtre et de son encrassement en surface, une partie de l'énergie des particules alpha est cédée au milieu et ne permet plus leur discrimination et leur détection par les compteurs proportionnels à mode simultané $\alpha / \beta$. Le rendement de mesure est alors dégradé et nécessite une correction de l'activité mesurée. Pour répondre aux conditions usuelles de mesure, le laboratoire a développé une modélisation globale permettant d'établir les facteurs de correction à prendre en fonction des paramètres de dépôt et des caractéristiques liées à la détection des radio-isotopes et de leur mesure. Cette approche a consisté à modéliser la proportion de particules alpha présentes dans le filtre qui est détectées en fonction de la masse surfacique d'aérosols collectés sur un filtre donné et des autres paramètres du problème.

L'évaluation des facteurs correctifs nécessite une connaissance la plus exhaustive possible des conditions d'expositions des filtres et des paramètres liés à leur mesure. L'accès à ces données est souvent difficile et a nécessité, dans notre cas, la prise en compte d'hypothèses :

* les caractéristiques physiques des aérosols sont supposées constantes au cours

du prélèvement, 
* le dépôt d'aérosols est considéré homogène sur la surface du filtre,

* les conditions d'hygroscopicité des filtres sont supposées constantes,

* les énergies initiales des particules alpha, émises par un même radionucléide, suivent une distribution gaussienne d'écart type $0,02 \mathrm{MeV}$,

* l'activité massique $a_{m}$ des aérosols retenus par le filtre est considérée constante et telle que la variation de l'activité déposée $d A$ soit proportionnelle à la variation de la masse d'aérosols déposés $d m$ :

$$
d A=a_{m} d_{m}
$$

La mesure de l'activité alpha des aérosols est intrinsèque aux conditions de prélèvement sur filtre. En fonction des mécanismes de dépôt, l'énergie cinétique des particules alpha est atténuée dans la matière qu'elles traversent et qui se dépose sur le filtre. L'étude entreprise a donc consisté à établir une relation entre les données de colmatage des filtres et le processus d'atténuation de l'énergie cinétique des particules alpha au cours de leur parcours vers le détecteur.

\subsection{Les modèles de colmatage des filtres plans}

La littérature (Del Fabbro, 2002) permet d'accéder aux différents modèles de colmatage des filtres plans à fibres. Nous citerons plus particulièrement les travaux de Pénicot et Thomas (Thomas et al., 1999, 2001), qui permettent de distinguer les phases de filtration en profondeur et en surface lorsque la masse surfacique d'aérosols déposée atteint une valeur critique intrinsèque aux caractéristiques du filtre et de l'aérosol. Leurs observations expérimentales permettent d'aboutir à différentes conclusions :

* la vitesse de filtration n'a pas d'influence sur la manière dont se déposent les particules, dans la gamme de vitesse étudiée (1 à $50 \mathrm{~cm} / \mathrm{s}$ ),

* les particules se répartissent de façon exponentiellement décroissante dans la profondeur du filtre d'épaisseur $e_{f i l t r e}$; la compacité des particules $\alpha_{p}$ à la profondeur $x$ est ainsi déduite par la relation :

$$
\alpha_{p}(x)=\left(\frac{m}{\Omega}\right)_{\text {filtre }} \frac{k e^{-k x}}{\rho_{\text {particules }}\left(1-e^{-k e_{\text {filtre }}}\right)}
$$

$(\mathrm{m} / \Omega)_{\text {filtre }}$ : masse d'aérosols collectée au sein du filtre par unité de surface $\left(\mathrm{kg} / \mathrm{m}^{2}\right), k$ : facteur de pénétration dans le filtre $\left(\mathrm{m}^{-1}\right), \rho_{\text {particules }}$ : masse volumique des particules $\left(\mathrm{kg} / \mathrm{m}^{3}\right), x$ : profondeur dans le filtre $(\mathrm{m})$,

* la compacité des particules au sein du gâteau $\alpha_{\text {gâteau }}$ est corrélée à leur diamètre $d_{p}($ en $\mu \mathrm{m})$ :

$$
\alpha_{\text {gâteau }}=0,58\left(1-\exp \left(-\frac{d_{p}}{0,53}\right)\right),
$$


* la masse surfacique critique dépend de la taille des particules, du diamètre des fibres et de la compacité du filtre.

Par corrélation aux valeurs expérimentales, différents modèles de perte de charge théoriques permettent d'accéder aux grandeurs caractéristiques de la filtration, par ailleurs nécessaires à l'élaboration d'une modélisation plus globale du facteur de correction de la mesure de l'activité alpha des aérosols.

\subsubsection{Modèle de Davies : (Davies, 1970)}

Ce modèle suppose que les particules d'aérosol se déposent uniformément à la surface de chaque fibre, entraînant une augmentation de leur diamètre et de la compacité du filtre. Il permet d'estimer le diamètre des fibres par corrélation de la perte de charge d'un filtre vierge avec les valeurs expérimentales :

$$
\Delta P_{\text {filtre vierge }}=\frac{16 \mu Q e_{\text {filtre }} \alpha_{\text {fibres }}^{\frac{3}{2}}}{\Omega r_{\text {fibres }}^{2}}\left(1+56 \alpha_{\text {fibres }}^{3}\right)
$$

$\mu$ : viscosité de l'air (Pa s), $e_{\text {filtre }}$ : épaisseur du filtre $(\mathrm{m}), \Omega$ : surface du filtre $\left(\mathrm{m}^{2}\right)$, $Q$ : débit d'air $\left(\mathrm{m}^{3} \mathrm{~s}^{-1}\right), \alpha_{\text {fibres }}$ : compacité des fibres $=(1-\varepsilon), \varepsilon:$ porosité du filtre, $r_{\text {fibres }}$ : rayon des fibres $(\mathrm{m}), \Delta P_{\text {filtre vierge }}$ : perte de charge du filtre vierge $(\mathrm{Pa})$.

\subsubsection{Modèle de Letourneau : (Letourneau et al., 1991)}

Inspiré du modèle de Bergman (Bergman et al., 1976), il permet de décrire la filtration en profondeur. Le modèle repose sur l'hypothèse d'une répartition exponentielle décroissante des aérosols dans le filtre et l'intégration du modèle de Bergman sur l'épaisseur du filtre :

$$
\begin{gathered}
\Delta P_{\text {filtre }}=\int_{0}^{e_{\text {filtre }}} 16 \mu \frac{Q}{\Omega}\left(\frac{\alpha_{\text {fibres }}}{r_{\text {fibres }}^{2}}+\frac{\alpha_{\text {particules }}(x)}{r_{\text {particules }}^{2}}\right)^{\frac{1}{2}}\left(\frac{\alpha_{\text {fibres }}}{r_{\text {fibres }}}+\frac{\alpha_{\text {particules }}(x)}{r_{\text {particules }}}\right) \\
\times\left(1+56\left(\alpha_{\text {fibres }}+\alpha_{\text {particules }}(x)\right)^{3}\right) d x
\end{gathered}
$$

$\alpha_{\text {particules }}(x)$ : compacité des particules à la profondeur $x$ dans le filtre (relation (2)), $r_{\text {particules }}$ : rayon des particules (m), $\Delta P_{\text {filtre }}$ : perte de charge du filtre $(\mathrm{Pa})$. 
2.2.3. Modèle de Novick : (Novick et al., 1992)

Novick assimile le dépôt de particules uniquement à la formation du gâteau. Pour des quantités élevées de particules, la perte de charge relative à la filtration en surface est considérée in facto comme la résultante de la composante liée au filtre vierge et de celle propre au gâteau $\left(\Delta P=\Delta P_{\text {filtre vierge }}+\Delta P_{\text {gâteau }}\right)$. La perte de charge du gâteau $\Delta P_{\text {gâteau }}$ est alors calculée selon la loi de Kozeny :

$$
\begin{gathered}
\Delta P_{\text {gâteau }}=\frac{h_{K} a_{g}^{2} \alpha_{\text {gâteau }}^{2} \mu Q e_{\text {gâteau }}}{\Omega C_{c}\left(1-\alpha_{\text {gâteau }}\right)^{3}} \\
e_{\text {gâteau }} \text { : épaisseur du gâteau }(\mathrm{m})=\frac{\frac{m_{\text {gâteauu }}}{\Omega}}{\alpha_{\text {gâteau }} \rho_{\text {particules }}} \\
\alpha_{\text {gâteau }} \text { : compacité des particules au sein du gâteau (relation (3)) } \\
a_{g}: \text { aire spécifique des particules }\left(\mathrm{m}^{-1}\right)=\frac{3}{r_{\text {particules }}}
\end{gathered}
$$

$m_{\text {gâteau }}$ : masse du gâteau, $h_{K}$ : constante de Kozeny-Carman (choisie égal à 5), $C_{c}$ : coefficient de Cunningham (choisi égal à 1), $\Delta P_{\text {gâteau }}$ : perte de charge du gâteau $(\mathrm{Pa})$.

La corrélation des valeurs expérimentales aux modèles de Letourneau et de Novick est obtenue après optimisation par la méthode Newton-Raphson. Ceci permet d'estimer les valeurs théoriques des caractéristiques du filtre et des aérosols déposés (Fig. $5-\S 2.4$ ).

Par ailleurs, les résultats des travaux de Pénicot et Thomas (Thomas et al., 1999, 2001) permettent d'accéder à la répartition des aérosols sur le medium filtrant (relations (2) et (3)). Ils montrent que la phase de filtration en surface survient quand la masse surfacique devient supérieure à une valeur critique qui dépend de la taille des particules, du diamètre des fibres et de la compacité du filtre.

Dans l'hypothèse d'une répartition uniforme de l'activité dans l'aérosol, l'interaction des particules alpha avec leur environnement (fibres du filtre et poussières collectées) peut alors être associée à la perte d'énergie linéique de ces particules pour l'évaluation de leur parcours vers un détecteur (Mulhauser, 2001; Yao et al., 2006). En fonction de leur parcours, la perte d'énergie cédée au milieu ne permet plus leur discrimination et donc leur détection par le système de mesure. La notion de «profondeur limite de détectabilité » a donc été introduite. 


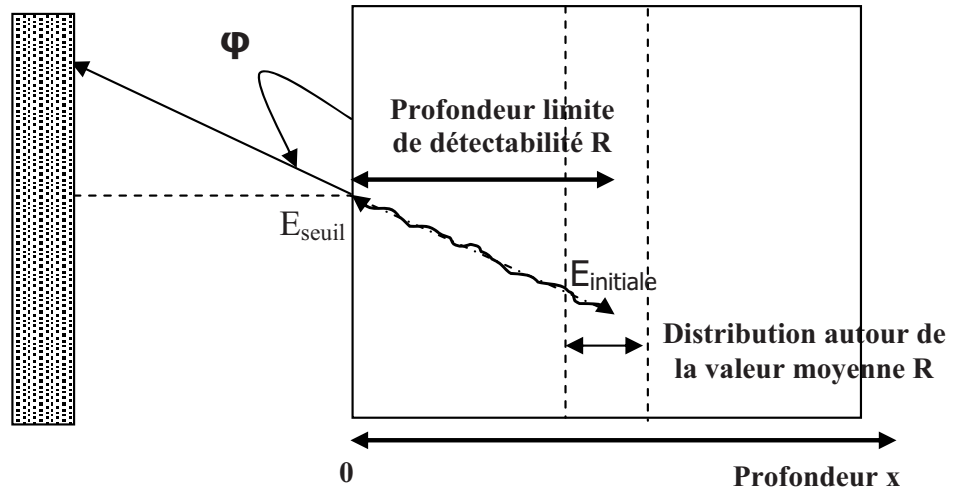

DETECTEUR

FILTRE + GÂTEAU

Figure 1 - Profondeur limite de détectabilité $R$ et sa distribution d'écart type $\sigma$.

Depth limit of detectability $R$ and its distribution (standard deviation $\sigma$ ).

\subsection{La détection et la mesure des particules alpha}

\subsubsection{Profondeur limite de détectabilité $R$}

La première étape de la modélisation consiste à estimer la profondeur limite de détectabilité $R(\varphi)$ (Fig. 1). Elle est définie comme la profondeur d'origine moyenne à partir de laquelle les particules alpha, traversant le matériau sous une certaine inclinaison $\varphi$ (exprimé en radian), ne sont plus détectées :

$$
R(\varphi)=\sin (\varphi) \int_{E_{\text {initiale }}}^{E_{\text {seuil }}}\left(\frac{d E}{d x}\right)^{-1} d E
$$

$R(\varphi)$ : Profondeur limite de détectabilité $(\mathrm{cm}),(d E / d x)$ : perte d'énergie linéique des particules alpha $=$ pouvoir d'arrêt du milieu traversé $\left(\mathrm{MeV} \mathrm{cm}^{-1}\right), E_{\text {initiale }}$ : énergie initiale des particules alpha $(\mathrm{MeV}), E_{\text {seuil }}$ : énergie à partir de laquelle les particules alpha ne sont plus détectées $(\mathrm{MeV})$.

La perte d'énergie linéique des particules alpha dans le milieu traversé (gâteau ou filtre) est extrapolée à partir de la loi de Bragg qui permet d'estimer le pouvoir d'arrêt du milieu traversé à partir de la fraction volumique $\alpha_{i}$ des différents matériaux le constituant et de leur pouvoir d'arrêt respectif (ICRU, 1993) :

$\left(\frac{d E}{d x}\right)_{\text {milieu traversé }}=\alpha_{\text {fibres }}\left(\frac{d E}{d x}\right)_{\text {fibres }}+\alpha_{\text {aérosols }}\left(\frac{d E}{d x}\right)_{\text {aérosols }}+\alpha_{\text {air }}\left(\frac{d E}{d x}\right)_{\text {air }}$

$\alpha_{\text {air }}=\varepsilon$ : porosité du filtre (sans dimension). 


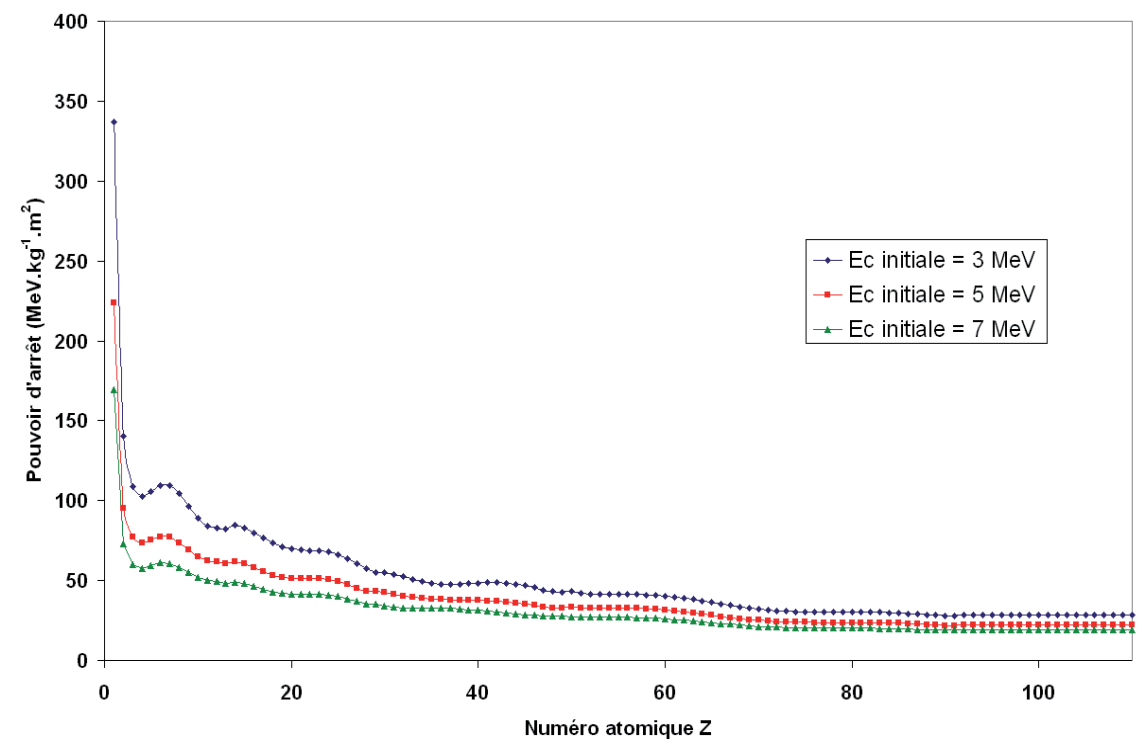

Figure 2-Pouvoir d'arrêt massique d'un élément en fonction de son numéro atomique Z pour différentes valeurs d'énergie cinétique initiale des particules alpha.

Nuclear stopping power versus the atomic number $Z$ and values of initial kinetic energy of alpha particles.

Le pouvoir d'arrêt massique $\left(\frac{d E / d x}{\rho}\right)$ (exprimé en $\mathrm{MeV} \mathrm{g}^{-1} \mathrm{~cm}^{2}$ ) des aérosols et des éléments constitutifs du medium filtrant sont extraits de la base de données ASTAR (ICRU, 1993) publiée par le National Institute of Standards and Technology (NIST).

À défaut, les pouvoirs d'arrêt massiques des éléments sont estimés à partir de leur numéro atomique (Fig. 2) et ceux des composés grâce à une variante de la loi de Bragg :

$$
\left(\frac{d E / d x}{\rho}\right)_{\text {composé }}=\sum \omega_{i}\left(\frac{d E / d x}{\rho}\right)_{\text {élément } i}
$$

$\omega_{i}$ : fraction massique en élément $i, \rho$ : masse volumique $\left(\mathrm{g} \mathrm{cm}^{-3}\right)$.

La valeur de profondeur limite est atteinte lorsque les particules alpha ressortent du filtre avec une énergie inférieure à l'énergie de discrimination de l'appareil de comptage en mode simultané $\alpha / \beta$ (généralement, $E_{\text {seuil }}<3 \mathrm{MeV}$ ). En raison de la nature statistique des collisions, la profondeur limite de détectabilité 


\section{Proportion de particules alpha détectées}

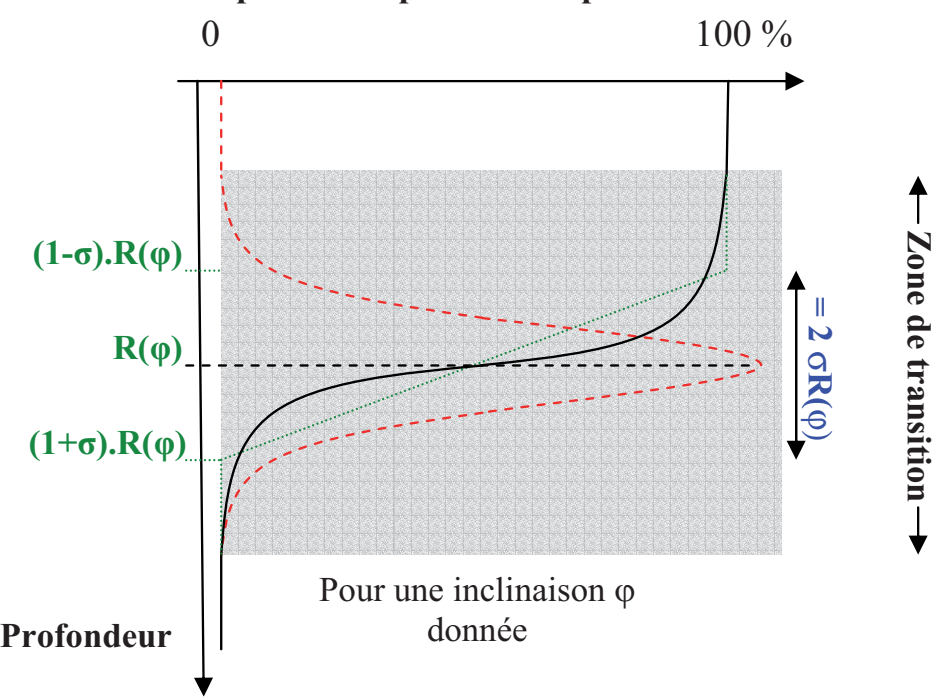

Figure 3 - Proportion de particules alpha détectées en fonction de leur profondeur d'origine.

Proportion of alpha particles detected versus origin depth.

fluctue autour de la valeur calculée selon une loi normale centrée sur $R(\varphi)$ et d'écart type $\sigma$ faible $(\sigma<1 \%)$ (Fig. 3).

La connaissance de $R(\varphi)$ permet d'accéder au taux de détection des particules alpha en fonction de leur profondeur d'origine.

\subsubsection{Le taux de détection}

Le taux de détection $T_{\alpha}(x, \varphi)$ correspond au ratio du nombre de particules alpha détectées $d A_{\text {détectée }}(x, \varphi)(\mathrm{en} \mathrm{Bq})$ provenant de la profondeur $x$ et d'angle $\varphi$ à la quantité de particules alpha $d A(x, \varphi)(\mathrm{en} \mathrm{Bq})$ émise par la source dans les mêmes conditions :

$$
T_{\alpha}(x, \varphi)=\frac{d A_{\text {détectée }}(x, \varphi)}{d A(x, \varphi)}=\frac{A_{0}}{N_{0}} \frac{d N(x, \varphi)}{d A(x, \varphi)}
$$

$A_{0}$ : activité de la source étalon de référence (Bq), $N_{0}$ : comptage net de la source étalon de référence (cps), $d N$ : comptage associé aux particules alpha provenant de la profondeur $x$ suivant l'angle d'inclinaison $\varphi$ (cps).

L'évolution du taux de détection en fonction de la profondeur est déterminée à partir de la profondeur limite de détectabilité $R(\varphi)$ et de son écart type $\sigma$ (Fig. 3). 
La quantité de particules alpha détectée correspond au nombre de particules alpha émergentes du filtre dont l'énergie est supérieure au seuil de discrimination de l'appareil de mesure.

Par construction, $T_{\alpha}(x, \varphi)$ décroît linéairement entre la profondeur limite minimum $(1-\sigma) R(\varphi)$ et la profondeur limite maximum $(1+\sigma) R(\varphi)$.

\subsubsection{Détermination du facteur de correction de mesure : $\boldsymbol{F}_{\alpha}$}

La mesure de l'activité alpha déposée sur le filtre nécessite une correction sur le rendement de détection pour tenir compte des particules alpha qui ont perdu trop d'énergie au cours de leur parcours dans le medium filtrant et qui ne sont alors pas détectées. Dans le cas général d'une mesure, l'activité déposée sur le filtre $A$ est déterminée par référence à une source d'étalonnage d'activité $A_{0}$ mesurée dans les mêmes conditions (CETAMA, 1973) :

$$
A=A_{0} \frac{N}{N_{0}} \frac{G_{0}}{G} \frac{\rho_{0}}{\rho} \Pi_{i=1}^{6} \frac{K_{0}^{i}}{K^{i}} \approx A_{0} \frac{N}{N_{0}} F_{\alpha}
$$

$N, N_{0}$ : comptage net respectivement du filtre échantillon et de la source étalon (cps), $K^{i}, K_{0}{ }^{i}$ : facteurs de correction de mesures du filtre échantillon et de la source étalon (corrections de rétrodiffusion et de diffusion, d'absorption dans le milieu situé entre la source et le détecteur, dans le filtre et dans la fenêtre du détecteur, et correction liée au schéma de désintégration), $\rho, \rho_{0}$ : rendement de l'ensemble de comptage, $G, G_{\mathrm{O}}$ : facteurs de géométrie utilisés pour les mesures respectives du filtre échantillon et de la source étalon, $F_{\alpha}$ : facteur de correction lié à l'atténuation des particules alpha dans le filtre en considérant des conditions de mesures identiques entre le filtre échantillon et la source étalon.

La détermination du facteur de correction $F_{\alpha}$ repose sur la combinaison des paramètres liés aux conditions d'exposition des filtres et de dégradation de l'énergie des particules alpha lors de leur parcours en direction du détecteur. Une première approche consiste à assimiler le filtre et son gâteau à une succession de cylindre d'épaisseur $d x$ (Fig. 4).

Dans ces conditions et dans l'hypothèse d'une distribution homogène des particules alpha dans l'aérosol, le taux de comptage est défini comme la somme des contributions $d N(x, \varphi, \theta)$ provenant des cylindres unitaires à la profondeur $x$ selon des angles $\varphi$ et $\theta$ :

$$
N=\int_{0}^{e_{\text {filtre }}+e_{\text {gâteau }}} \int_{-\pi / 2}^{\pi / 2} \int_{0}^{2 \pi} d N(x, \varphi, \theta)
$$

$r_{\text {détecteur }}:$ rayon du détecteur $(\mathrm{m}), r_{\text {filtre }}$ : rayon utile du filtre $(\mathrm{m}), d_{\text {filtre-détecteur }}$ : distance entre le filtre et le détecteur $(\mathrm{m})$, Aire 1 : aire de la partie gauche de 


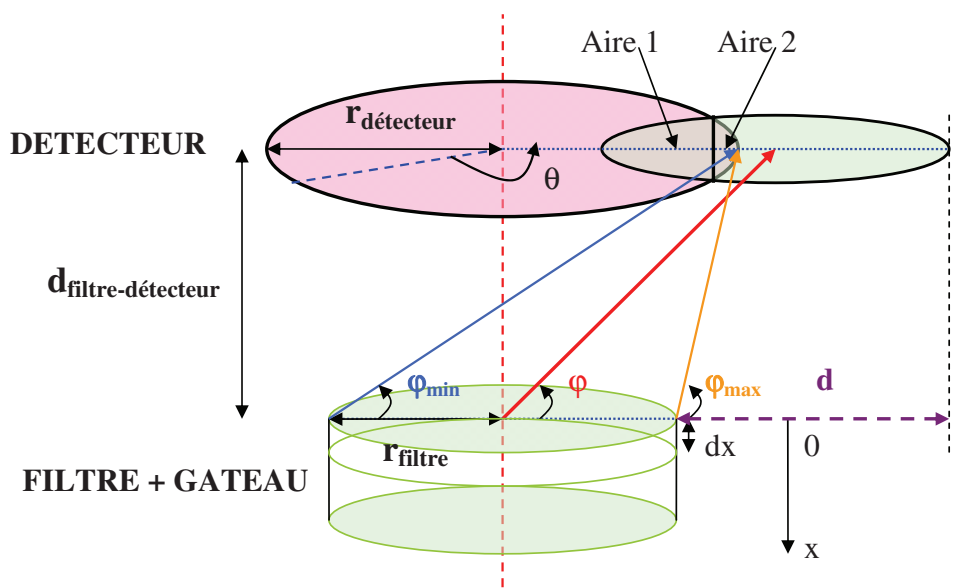

Figure 4 - Représentation schématique de l'ensemble filtre-détecteur dans le cadre du modèle. Schematic representation of the filter-detector system.

l'intersection entre la surface du détecteur et la projection de la surface du filtre $\left(\mathrm{m}^{2}\right)$, Aire 2 : aire de la partie droite de l'intersection entre la surface du détecteur et la projection de la surface du filtre $\left(\mathrm{m}^{2}\right)$

Aire $1=$
$\frac{r_{\text {flitre }}^{2} d^{2}\left(2 \pi-4 \arcsin \left(\frac{r_{\text {flitre }}^{2}-r_{\text {detecteur }}^{2}-d^{2}}{2 r_{\text {flitre }}}\right)\right)-\left(r_{\text {flitre }}^{2}-r_{\text {detecteur }}^{2}+d^{2}\right) \sqrt{-r_{\text {flltre }}^{4}+2 r_{\text {flitre }}^{2}\left(r_{\text {detecteur }}^{2}+d^{2}\right)-\left(r_{\text {detecteur }}^{2}-d^{2}\right)^{2}}}{4 d^{2}}$

Aire $2=$

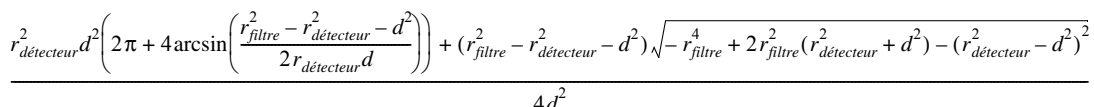

$$
d=\frac{d_{\text {filtre }- \text { détecteur }}}{\tan \varphi} .
$$

En introduisant la notion de taux de détection $T_{\alpha}$, la relation exprimant le taux de comptage $N$ devient :

$$
N=\int_{0}^{e_{\text {filtre }}+e_{\text {gâteau }}} \int_{-\pi / 2}^{\pi / 2} \int_{0}^{2 \pi}\left(\frac{N_{0}}{A_{0}}\right) T_{\alpha}(x, \varphi, \theta) d A(x, \varphi, \theta) .
$$

Appliqué aux conditions de prélèvements et de mesures des aérosols sur le filtre et en considérant que l'épaisseur du filtre et du gâteau sont négligeables par rapport 
à la distance filtre-détecteur, l'expression du taux de comptage s'écrit :

$$
N=\left(\frac{N_{0}}{A_{0}}\right)^{e_{\text {filtre }}} \int_{0}^{+e_{\text {gateau }} \pi / 2} \int_{\varphi_{\min }}^{\pi / 2} T_{\alpha}(x, \varphi) d A(x) \bar{\omega}(\varphi) \frac{d \varphi}{\psi}
$$

$d A(x)$ : activité présente dans la couche à la profondeur $x$. Selon les hypothèses formulées :

$$
\begin{gathered}
d A(x)=a_{m} \Omega \rho_{p} \alpha_{p}(x) d x(\mathrm{~Bq}) \\
\varphi_{\max }=\arctan \left(\frac{d_{\text {filtre }- \text { détecteur }}}{\left|r_{\text {détecteur }}-r_{\text {filtre }}\right|}\right) \text { (radian) } \\
\varphi_{\min }=\arctan \left(\frac{d_{\text {filtre }- \text { détecteur }}}{\left|r_{\text {détecteur }}-r_{\text {filtre }}\right|}\right) \text { (radian) }
\end{gathered}
$$

$\varpi(\varphi)$ : facteur de correction géométrique $=$ aire de l'intersection.

$$
\begin{aligned}
& \varpi=\frac{\text { Aire }_{1}+\text { Aire }_{2}}{\Pi r_{\text {filtre }}^{2}}\left(\text { pour } \varphi_{\min } \leq \varphi \leq \varphi_{\max }\right) \\
& \left.\bar{\omega}=1 \text { (pour } \varphi_{\max } \leq \varphi \leq \pi / 2 \text { et } r_{\text {détecteur }} \geq r_{\text {filtre }}\right) \\
& \left.\varpi=\left(\frac{r_{\text {détecteur }}}{r_{\text {filtre }}}\right)^{2} \text { (pour } \varphi_{\max } \leq \varphi \leq \pi / 2 \text { et } r_{\text {détecteur }} \leq r_{\text {filtre }}\right) \\
& \Psi: \text { facteur de normalisation (radian) }=\int_{\varphi_{\min }}^{n / 2} \varpi(\varphi) d \varphi \\
& e_{\text {gâteau }} \text { : épaisseur du gâteau (m) }=\frac{m_{\text {gâteau }}}{\Omega \rho_{\text {particules }} \alpha_{\text {gâteau }}} \\
& e_{\text {filtre }} \text { : épaisseur du filtre }(\mathrm{m}) .
\end{aligned}
$$

Ainsi, le facteur de correction $F_{\alpha}$ peut être corrélé à la masse surfacique d'aérosol déposée $m / \Omega\left(\mathrm{kg} \mathrm{m}^{-2}\right)$ :

$$
F_{\alpha}=\frac{N_{0}}{N} \frac{A}{A_{0}}=\frac{N_{0}}{N} \frac{a_{m} m}{A_{0}}=\frac{(m / \Omega)}{\rho_{p} \int_{0}^{e_{\text {filtre }}+e_{\text {gateau }} \pi / 2} \int_{\varphi_{\min }}^{\pi} T_{\alpha}(x, \varphi) \alpha_{p}(x) d x \varpi(\varphi) \frac{d \varphi}{\psi}}
$$


$\alpha_{p}(x)$ : compacité des particules à la profondeur $x(\mathrm{~m})$ dans le filtre (relation (2)), $\rho_{p}$ : masse volumique des particules $\left(\mathrm{kg} \mathrm{m}^{-3}\right), N, N_{0}$ : taux de comptage net respectivement du filtre échantillon et de la source étalon de référence (cps), $A, A_{0}$ : Activité respectivement du filtre échantillon et de la source étalon de référence $(\mathrm{Bq}), a_{m}$ : activité massique des particules $\left(\mathrm{Bq} \mathrm{kg}^{-1}\right)$.

\subsection{Approche expérimentale}

La vérification du modèle pour l'évaluation du facteur de correction de mesure $F_{\alpha}$ est réalisée à partir d'un gel de silice commercial calibré 5-20 $\mu \mathrm{m}$ (LiChroprep Si $60^{\circledR}$ - Fournisseur: Merck $^{\circledR}$ ) déposé sur un filtre à fibre de verre C357 de diamètre utile $130 \mathrm{~mm}$ (fournisseur - B.DUMAS). Le dépôt est effectué sur une Balise à Filtre Séquentiel Alpha/Bêta (BFSAB) par filtration de 10 à $60 \mathrm{~m}^{3}$ d'air chargé de gel de silice (débit nominal de prélèvement $\approx 60 \mathrm{Nm}^{3} / \mathrm{h}$ ). La mesure d'activité est réalisée à partir d'un compteur proportionnel fonctionnant en mode simultané $\alpha / \beta$ (Compteur PEGASE - CANBERRA - Fenêtre IN500 $\varnothing 135$ mm seuil de discrimination $<3 \mathrm{MeV})$.

\subsection{1. Évaluation des caractéristiques de dépôt}

Pour accéder à la valeur de différentes caractéristiques de la filtration, le laboratoire a procédé par corrélation et optimisation des modèles aux valeurs expérimentales de pertes de charge (Fig. 5). L'objectif recherché vise à évaluer l'ensemble des données nécessaires pour une estimation plus globale du facteur de correction de mesure de l'activité alpha des aérosols ; la détermination exacte et exhaustive des caractéristiques de filtration n'a donc pas été recherchée.

\subsubsection{Mesure du facteur de correction}

La contamination homogène du filtre est obtenue par filtration d'un gel de silice (LiChroprep Si $60^{\circledR}$ - Fournisseur: Merck ${ }^{\circledR}$ ) imprégné de tri-n-laurylamine permettant la stabilisation du ${ }^{239} \mathrm{Pu}$ (addition fractionnée en amont du dispositif de filtration à raison de 1 à $5 \mathrm{mg}$ de gel par $\mathrm{m}^{3}$ d'air filtré). L'activité en plutonium déposée sur la silice est supposée homogène et égale à $115 \pm 25 \mathrm{~Bq} / g_{\mathrm{SiO}_{2}}(k=2)$.

La mesure de l'activité en ${ }^{239} \mathrm{Pu}$ est intrinsèque aux conditions de dépôt de la silice sur le filtre (Fig. 6) ; dans une première étape, il y a enfouissement de la silice dans le filtre et dégradation de la mesure par interaction des particules alpha dans le filtre, qui explique la hausse du facteur de correction.

Quand la quantité de silice déposée devient supérieure à la masse surfacique critique, les particules de $\mathrm{SiO}_{2}$ commencent à se déposer à la surface du filtre. La 


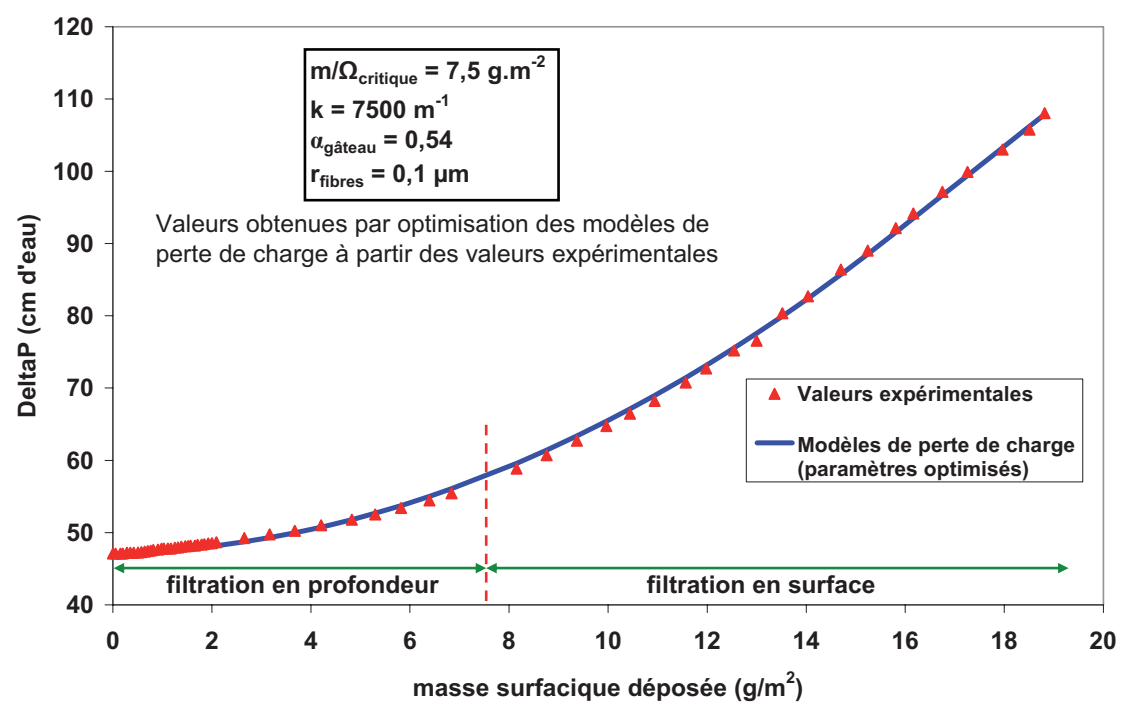

Figure 5 - Évolution de la perte de charge en fonction de la masse surfacique (Filtre C357 - Silice 5-20 $\mathrm{\mu m}$ ).

Pressure loss versus silica gel mass (Filter C357 - Silica gel 5-20 $\mu \mathrm{m}$ ).

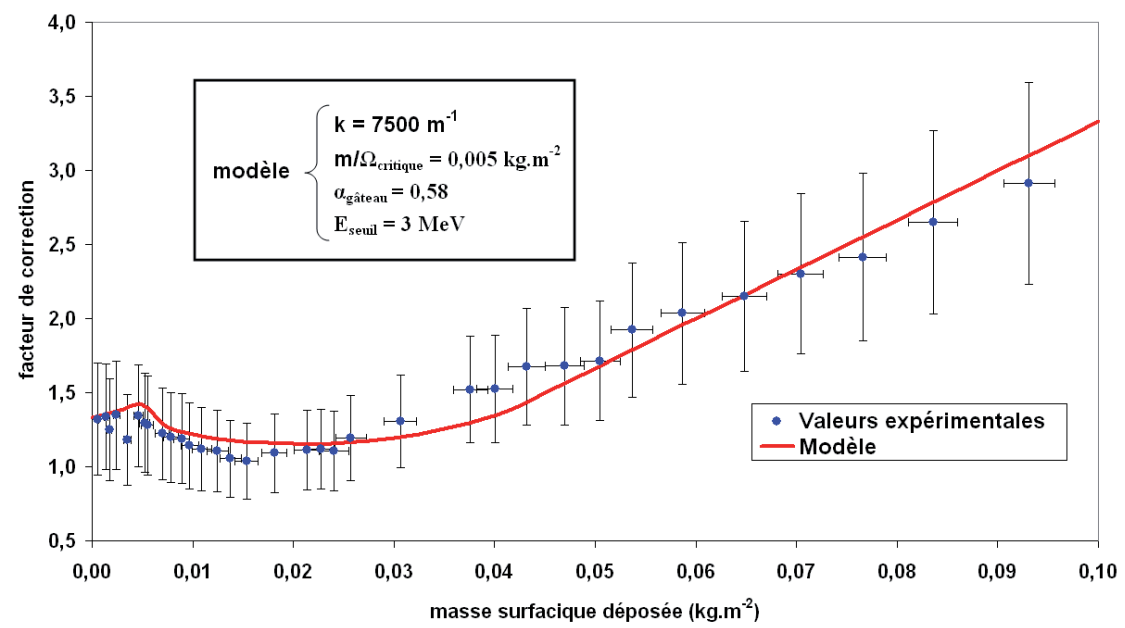

Figure 6 - Évolution et modélisation du facteur de correction de mesure avec la masse de silice déposée (Optimisation des paramètres de modélisation par la méthode de Newton-Raphson).

Experimental variation of the alpha activity correction factor versus silica gel mass on the filter-Optimization of the calculation parameters (Newton-Raphson method). 
mesure est alors très peu impactée par le dépôt et traduit une diminution du facteur de correction.

Enfin, lorsque l'épaisseur du gâteau devient supérieure à la profondeur limite de détectabilité, l'activité des premières couches de gâteau n'est plus détectée et le facteur de correction devient proportionnel à la quantité de silice déposée.

L'interpolation numérique appliquée aux mesures de l'activité vérifie le modèle pour l'évaluation de $F_{\alpha}$ (Fig. 6). Compte tenu des incertitudes de mesures (supérieures à $40 \%$ à $k=2$ ) et des difficultés pour la détermination des paramètres de filtration ( $k$ en particulier), la corrélation des valeurs expérimentales au modèle est enveloppe. Une prospective expérimentale permettant un encadrement fidèle des caractéristiques de filtration $\left(k, r_{\text {fibre }}, m / \Omega_{\text {critique }}, \alpha_{g} \ldots\right)$ et de mesure $\left(E_{\text {seuil }}\right)$ apporterait les éléments nécessaires à une évaluation serrée de $F_{\alpha}$ en fonction de la masse d'aérosols déposée ; cette démarche théorique serait néanmoins difficile à appliquer en routine compte tenu de la variété des aérosols collectés dans l'environnement, de la nature des radionucléides mesurés et des conditions d'exposition des filtres. La variabilité de $F_{\alpha}$ en fonction des différents paramètres de rétention et de mesure a donc été simulée afin d'évaluer leur contribution à l'incertitude finale de mesure.

\subsubsection{Influence de la nature de l'aérosol sur $\boldsymbol{F}_{\alpha}$}

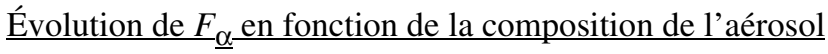

Les aérosols atmosphériques sont un mélange complexe de particules de différentes natures: suies, matière organique particulaire (MOP), poussières minérales (PM), débris métalliques et sels plus ou moins complexes. Leur composition très hétérogène modifie les conditions de dépôt et de parcours des particules alpha en fonction des densités des particules collectées.

La composition chimique moyenne des aérosols prélevés en région parisienne est fournie par AIRPARIF (2006) et permet d'accéder aux profils des corrections de mesure d'activité présentés en figure 7.

Les prélèvements effectués dans les stations de surveillance de l'environnement du CEA de Fontenay aux Roses sont réalisés sur 24 heures à partir d'une BFSAB. La masse surfacique d'aérosols déposée sur le filtre est inférieure à $3 \times 10^{-3} \mathrm{~kg} \mathrm{~m}^{-2}$ (valeur vérifiée par échantillonnage sur une année de collecte) ou encore à $(\mathrm{m} / \Omega)_{\text {critique. Dans les conditions d'une filtration en }}$ profondeur $\left((\mathrm{m} / \Omega)_{\text {critique }}\right.$ non atteinte et faible), la composition de l'aérosol n'influe pas sur la correction de mesure $\left(\Delta F_{\alpha}<5 \%\right)$. 


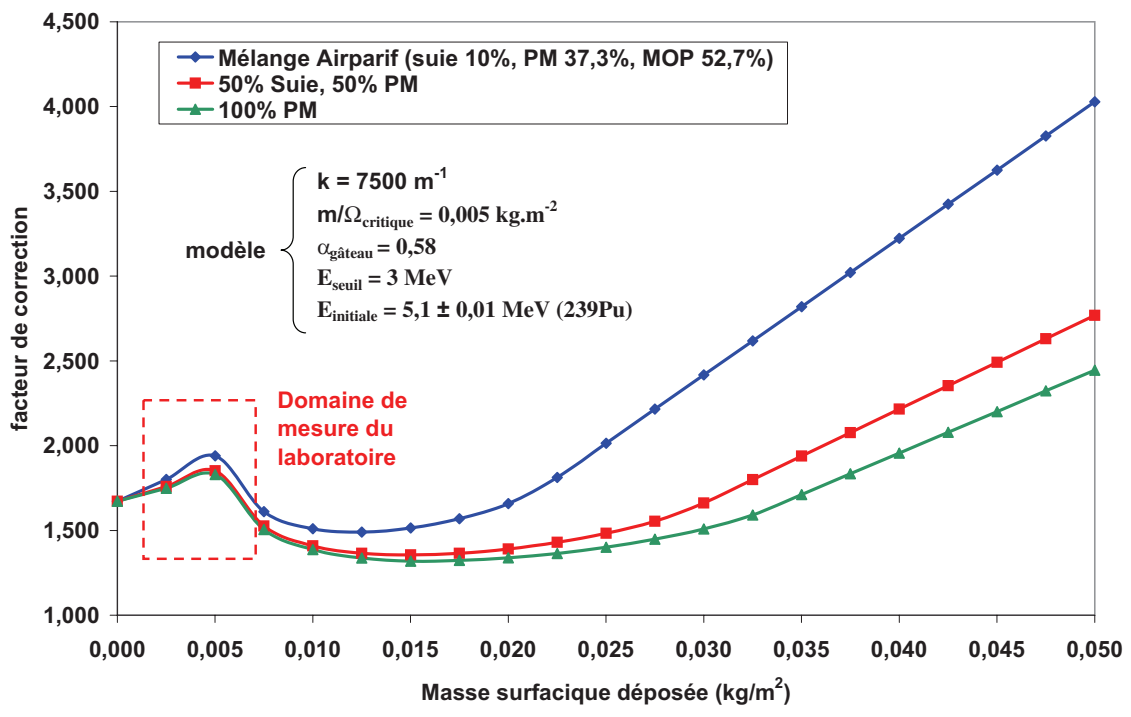

Figure 7 - Influence de la nature de l'aérosol sur le facteur de correction de la mesure d'activité alpha. Influence of the aerosol composition on the alpha activity measurement correction.

\section{Évolution de $\mathrm{F}_{\alpha}$ en fonction de la granulométrie de l'aérosol}

Le calcul de $F_{\alpha}$ repose sur l'hypothèse d'une distribution exponentielle décroissante des particules dans le filtre (Thomas et al., 1999, 2001). L'évolution de cette répartition varie avec la granulométrie des aérosols selon le facteur de pénétration $k$ pour la phase de filtration en profondeur (Fig. 8). Avec la formation d'un dépôt en surface, un écart de plusieurs $\mu \mathrm{m}$ sur le diamètre des particules formant un aérosol élémentaire $\left(\mathrm{SiO}_{2}\right.$ par exemple) ne modifie pas la valeur de $F_{\alpha}$ (pour une compacité de gâteau $\alpha_{g}>0,08$ ).

La masse surfacique critique est intrinsèque aux caractéristiques physiques de l'aérosol (granulométrie, composition,...); elle augmente avec la taille des particules (Fig. 9) et la densité des composés formant l'aérosol.

Le contrôle de l'activité des aérosols prélevés dans les stations de surveillance de l'environnement est réalisé à partir de dépôts présentant une grande diversité de particules en taille et en composition chimique (AIRPARIF, 2006). Compte tenu des sources d'émission et des processus de formation des aérosols, les caractéristiques des particules déposées ne sont pas directement accessibles et ne permettent pas le calcul de la valeur vraie de $F_{\alpha}$ dans le cadre d'une mesure de 


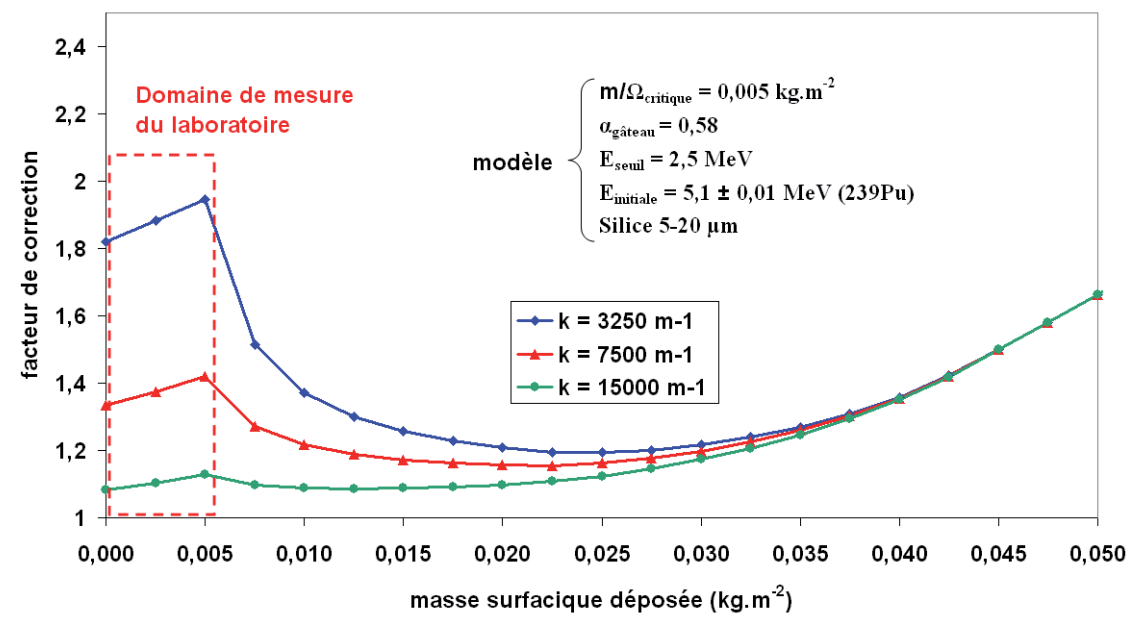

Figure 8 - Influence du facteur de pénétration $k$ sur le facteur de correction de la mesure d'activité alpha. Influence of the penetration factor ( $k$ ) on the alpha activity measurement correction.

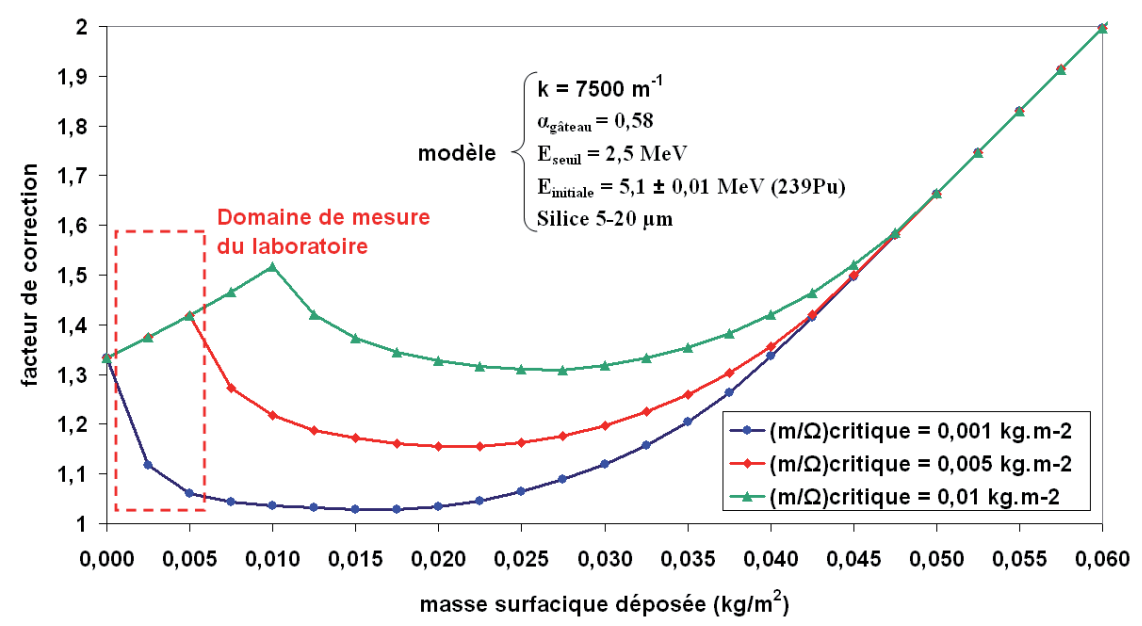

Figure 9 - Influence de la masse surfacique critique sur le facteur de correction de la mesure d'activité alpha.

Influence of the aerosol mass on the alpha activity measurement correction. 


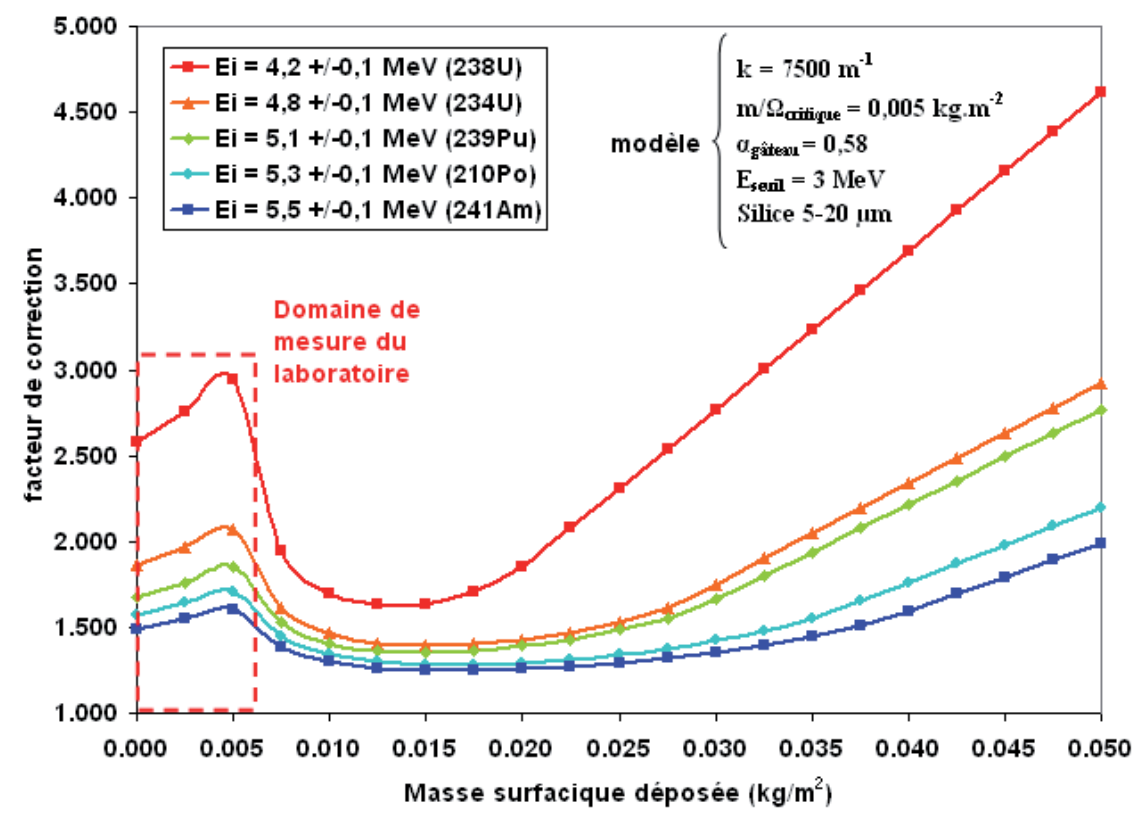

Figure 10-Influence de l'énergie des particules alpha sur le facteur de correction de la mesure d'activité.

Influence of the alpha particle energy on the activity measurement correction.

surveillance. L'étude de la variabilité de $F_{\alpha}$ rapportée aux conditions courantes de prélèvements observés par le laboratoire montre des écarts jusqu'à $80 \%$ avec le facteur de pénétration $k$ (Fig. 8) et avec $(m / \Omega)_{\text {critique }}$ (Fig. 9). L'utilisation d'un filtre à membrane téflon (FSLW) permettrait de limiter le prélèvement de l'aérosol à un dépôt de surface et diminuerait in facto la contribution liée à la nature des particules sur le calcul de $F_{\alpha}$ (Grivaud et Pagliardini, 2004).

\subsubsection{Influence de l'énergie des particules alpha}

Évolution de $\mathrm{F}_{\alpha} \underline{\text { en fonction de l'énergie des particules alpha }}$

La détection des particules alpha à partir d'un compteur proportionnel à mode simultané $\alpha / \beta$ est réalisée par sélection d'amplitude sur les impulsions issues du détecteur. Les amplitudes des impulsions sont proportionnelles aux énergies délivrées par les radionucléides déposés sur le filtre. En fonction de l'énergie cédée au milieu, l'énergie de la particule alpha ne suffira plus suffisante pour sa détection $\left(E_{\text {seuil }}\right)$ et se traduira par une dégradation du rendement de mesure. Selon les énergies des radionucléides émetteurs alpha, la correction de mesure sera différente et peut atteindre des écarts importants $\left(\Delta F_{\alpha}>50 \%\right)$ (Fig. 10). 
L'essentiel de la radioactivité déposée sur les filtres est attribuable à la radioactivité naturelle $\left({ }^{222} \mathrm{Rn}\right.$ et ses descendants de période longue tel que le ${ }^{210} \mathrm{Po}$ ). En cas de variation anormale de l'activité, les aérosols d'origine artificielle sont recherchés par le laboratoire et concernent le ${ }^{241} \mathrm{Am}$ et ${ }^{239} \mathrm{Pu}$, par ailleurs utilisé pour l'étalonnage en rendement du compteur. Le domaine d'énergie couvert par les radionucléides mesurés est important et se traduit par des écarts sur $F_{\alpha}$ supérieurs à $25 \%$.

\section{Conclusion et prospectives}

S'inscrivant dans le cadre de la surveillance de l'environnement, la mesure de la radioactivité des aérosols atmosphériques est confrontée à de nombreuses difficultés pour la détermination de l'activité déposée; en fonction des mécanismes de prélèvement sur filtre et des paramètres liés à la détection des radio-isotopes et de leur mesure, l'activité des émetteurs alpha est sous estimée et nécessite une correction de rendement. Cette correction est difficile d'accès et nécessite l'utilisation d'outils de calcul empirique pour son évaluation. Pour répondre aux conditions usuelles de mesure, le laboratoire a donc développé une modélisation globale permettant d'établir les facteurs de correction en fonction de la masse surfacique des aérosols collectés sur un filtre donné.

Compte tenu de la diversité des facteurs pouvant dégrader la qualité de la mesure, le calcul systématique de cette correction n'a cependant pas été recherché. La démarche adoptée a consisté à évaluer les contributions des conditions de prélèvement et de mesure sur l'incertitude finale du résultat d'activité. La variabilité de $F_{\alpha}$ en fonction des différents paramètres de rétention et de mesure a donc été simulée afin d'estimer les écarts sur la mesure d'activité que l'on pourrait observer à partir d'une valeur de correction moyenne.

Cette étude a été menée indépendamment des relations entre chaque paramètre de filtration et de mesure; dans les conditions habituelles de prélèvement du laboratoire $\left(\mathrm{m} / \Omega \approx 3 \times 10^{-3} \mathrm{~kg} / \mathrm{m}^{3}\right.$, débit de prélèvement $\approx 60 \mathrm{~m}^{3} / \mathrm{h}$, filtre à fibre de verre $\mathrm{C} 357$ ), elle a conduit à une première estimation du facteur de correction autour de 1,4 pour la mesure de la radioactivité d'origine naturelle. Les valeurs enveloppe de $F_{\alpha}$ respectivement 1 et 2 confirment les études précédentes (Grivaud et Pagliardini, 2004) et représentent la principale contribution à l'incertitude globale de la mesure d'activité.

Les principales sources de variation de $F_{\alpha}$ proviennent des caractéristiques physiques de l'aérosol, dont l'influence est particulièrement importante pour une filtration en profondeur ; une forte amplitude de pénétration des particules dans le filtre se traduit par des écarts importants $\operatorname{sur} F_{\alpha}\left(\Delta F_{\alpha} \approx 80 \%\right.$ pour $k$ compris entre 
5000 et 120000 dans les conditions de l'étude). Compte tenu de la diversité des aérosols prélevés dans l'environnement, l'utilisation d'un facteur de correction de mesure moyen a été retenu par le laboratoire afin de couvrir la gamme des aérosols susceptibles d'être prélevés en région parisienne $\left(F_{\alpha}^{l a b o}=1,6 \pm 0,5\right)$.

Au regard des résultats obtenus, l'étude a permis de valoriser la portée de la mesure de l'indice d'activité alpha dont le fondement reste une mesure de contrôle de la qualité de notre environnement. En cas de variation importante de l'activité mesurée (au delà d'un facteur 2 par rapport à une valeur de référence), une investigation complémentaire est nécessaire.

\section{RÉFÉRENCES}

AIRPARIF (2006) Surveillance de la Qualité de l'Air en Ile-de-France, octobre 2006. Étude des retombées de suies sur les matériaux en sites peri-aéroportuaires, Comparaison avec des sites urbains et ruraux.

Bergman W., Taylor R.D., Miller H.H., Bierman A.H., Hebard H.D., Daroza R.A., Lum B.Y. (1976) Enhanced filtration program at L.L.L. progress report. Lawrence Livermore LaboratoryLivermore, California.

CETAMA (1973) Mesures d'activité nucléaire, Edition Masson.

Davies C.N. (1970) The clogging of fibrous aerosol filters, J. Aerosol Sci. 1, 35-39.

Del Fabbro L. (2002) Rapport CEA-R-6002. Modélisation des écoulements d'air et du colmatage des filtres plissés par des aérosols solides.

Grivaud L., Pagliardini F. (2004) Rapport d'essais IRSN/DSU/SERAC/LPMA/04 02, Mesure de la radioactivité alpha des aérosols prélevés sur filtres C357 - Etude de faisabilité.

ICRU (1993) Report 49, Stopping powers and ranges for protons and alpha particles.

Letourneau P., Renaudin V., Vendel J. (1991) Effects of the particle penetration inside the filter medium of the HEPA filter pressure drop. 22nd DOE Nuclear Air Cleaning Conference, Denver.

Mulhauser F. (2001) Université de Fribourg - Constituants de la matière - Interactions des rayonnements avec la matière. http://www.unifr.ch/physics/me/cours/methodes/script.html

Novick V.J., Monson P.R., Ellison P.G. (1992) The effect of solid particle mass loading on the pressure drop of HEPA filters, J. Aerosol Sci. 23, 657-665.

Thomas D., Contal P., Renaudin V., Penicot P., Leclerc D., Vendel J. (1999) Modelling pressure drop in HEPA filters during dynamic filtration, J. Aerosol Sci. 30, 235-246.

Thomas D., Pénicot P., Contal P., Leclerc D., Vendel J. (2001) Clogging of fibrous filters by solid aerosol particles. Experimental and modelling study, Chem. Engineer. Sci. 56, 3549-3561.

Yao W.-M. et al. (2006) Passage of particles through matter, J. Phys. G 33, 27. 